\title{
Age of red blood cells and transfusion in critically ill patients
}

\author{
Cécile Aubron ${ }^{*}$, Alistair Nichol, D Jamie Cooper and Rinaldo Bellomo
}

\begin{abstract}
Red blood cells (RBC) storage facilitates the supply of RBC to meet the clinical demand for transfusion and to avoid wastage. However, RBC storage is associated with adverse changes in erythrocytes and their preservation medium. These changes are responsible for functional alterations and for the accumulation of potentially injurious bioreactive substances. They also may have clinically harmful effects especially in critically ill patients. The clinical consequences of storage lesions, however, remain a matter of persistent controversy. Multiple retrospective, observational, and single-center studies have reported heterogeneous and conflicting findings about the effect of blood storage duration on morbidity and/or mortality in trauma, cardiac surgery, and intensive care unit patients. Describing the details of this controversy, this review not only summarizes the current literature but also highlights the equipoise that currently exists with regard to the use of short versus current standard (extended) storage duration red cells in critically ill patients and supports the need for large, randomized, controlled trials evaluating the clinical impact of transfusing fresh (short duration of storage) versus older (extended duration of storage) red cells in critically ill patients.
\end{abstract}

Keywords: Age of red blood cells, Storage lesion, Critically ill patients, Outcome, Transfusion, Anemia, Trauma, Cardiac surgery, Mortality, Cytokines

\section{Introduction}

Anemia is common in critically ill patients: up to $90 \%$ of patients will be anemic by day 3 of their intensive care unit (ICU) stay [1,2]. Red blood cells (RBC) transfusion rates in critically ill patients are reported between $20 \%$ and $40 \%$ in ICU [2-4], with a mean of 2 to 5 RBC units transfused per patient $[3,4]$. Such anemia of critical illness has been associated with a poor prognosis even in the absence of ischemic heart disease $[3,5,6]$. This association supports the value of RBC transfusion in critically ill patients. Nonetheless, although potentially life-saving for individual patients, RBC transfusion also has been associated with an increased risk of morbidity and/or mortality in critically ill, surgical, and trauma populations $[7,8]$. In this setting, studies have increasingly focused on the possible deleterious role played by RBC storage duration (so-called age of red cells) [9-12]. In particular, they have raised concerns that prolonged RBC storage may lead to harm once such "older" red cells are transfused into ICU patients.

To avoid wasting RBC units and improve the provision of blood stock, standard practice worldwide consists of transfusing the oldest compatible and available RBC unit. In addition, RBC can be stored up to 42 days maximizing their availability and the likelihood that red cells older than 2 weeks will be transfused into critically ill patients. This RBC storage duration usually up to 42 days has been defined on the bases of 1 ) a percentage of RBC still present in the circulation 24 hours after transfusion higher than $75 \%$, and 2) hemolysis $<1 \%$ at the end of the storage period [13]. Despite improved preservation methods, "storage lesions" occur in such cells, because in a way that increases over time erythrocytes develop important biochemical and structural derangements that affect their function and possibly their safety $[10,14]$.

\footnotetext{
* Correspondence: cecile.aubron@monash.edu

ANZIC Research Center, School of Public Health \& Preventive Medicine,

Monash University, The Alfred Center, 99 Commercial Road, Melbourne, VIC 3004, Australia
}

\section{黑 Springer}




\section{Current practice and concerns with "age of red cells" for ICU patients}

The mean age of blood transfused in ICU patients varies from 16 to 21 days and is very similar throughout the world [2,3]. A large range of adverse effects related to $\mathrm{RBC}$ storage have been reported in critically ill patients when RBC stored for 2 to 4 weeks are transfused. These include increased mortality [15-22], nosocomial infections [18,22-27], multiple organ failure [18,28], renal failure $[18,22]$ deep vein thrombosis [20], increase in ICU [15] and hospital length of stay (LOS) [29,30], and in mechanical ventilation duration [18]. Nonetheless, most clinical studies in this area have been observational in nature, retrospective in design, small in size, and subject to bias, leaving this issue unresolved for more than 20 years.

Given the above concerns, further research to determine the effect of storage lesion (age of red cells) on clinical outcome in critically ill patients seems important. If fresh blood is associated with a decrease of mortality in critically ill patients, changes in transfusion policies will be applied. Considering the cost and the potential consequences on blood supply of delivery of fresh blood instead old blood, pivotal trials are required to answer definitively whether age of blood impacts the mortality and or morbidity of critically ill patients.

This review will consider: 1) the nature of storage lesions and why critically ill patients may be especially susceptible to the adverse effects of prolonged RBC storage; 2) the experimental studies that have explored the impact of blood storage on tissue oxygenation parameters; 3 ) the clinical studies of the effect of prolonged storage in adult trauma, postcardiac surgery, or ICU patients; and 4) the need for randomized, controlled studies in this field.

\section{RBC storage lesions}

During storage, $\mathrm{RBC}$ and their preservative medium suffer metabolic, biochemical, and molecular changes commonly referred to as "storage lesion" (Figure 1; Table 1) $[10,31]$. Structural RBC changes include depletion of adenosine triphosphate (ATP) and of 2,3-diphosphoglycerate (2,3-DPG); membrane phospholipid vesiculation; protein oxidation; and lipid peroxidation of the cell membrane $[10,31,32]$. Over time, RBC shape changes with increased osmotic fragility and loss of deformability $[31,33]$. Decreased membrane flexibility may compromise the effect of RBC on microcirculatory flow and participates in increasing red cell-endothelial cell interaction, with activation of inflammatory pathways. Furthermore, bioreactive substances accumulate in storage medium. These include 1) lipids that prime recipient neutrophils and have been implicated in transfusion-related acute lung injury [34]; 2) cytokines; and 3) free iron from hemolysis [35].

Recently, a study conducted in healthy volunteers reported the presence of higher extravascular hemolysis after older RBC transfusion (storage of 40-42 days) compared with fresh blood (storage of 3-7 days) illustrating the possible harmful effect of iron delivery [36]. This RBC "storage lesion" also alters oxygen delivery (because of the higher affinity of hemoglobin to oxygen secondary to a decrease in 2,3-DPG) and $\mathrm{pH}$ and, in addition, it increases cell lysis [10]. Free hemoglobin

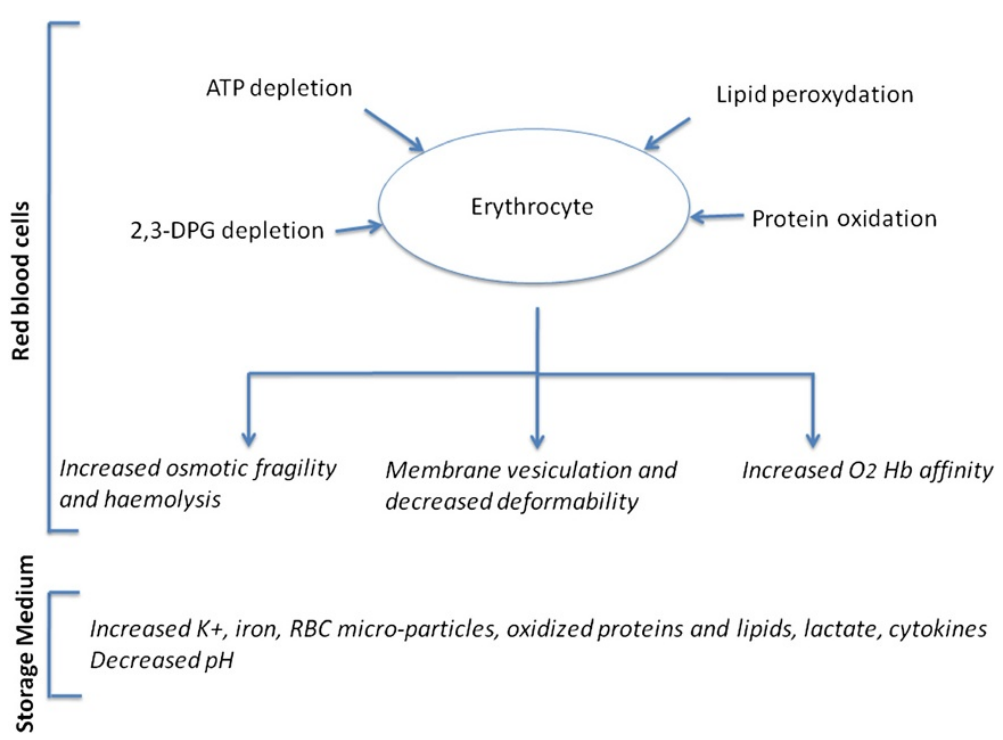

Figure 1 Changes occurring in red blood cells and storage medium over the storage time. ATP adenosine triphosphate; 2,3-DPG 2,3diphosphoglycerate; $R B C$ red blood cells. 
Table 1 Main biochemical changes in RBC storage medium and in loss of RBC deformability over the storage period $[10,67]$

\begin{tabular}{|c|c|c|c|c|}
\hline \multirow[t]{2}{*}{ Variables } & \multicolumn{4}{|c|}{ Length of storage } \\
\hline & Day 1 & Days 7 & Days 15 & Day 42 \\
\hline $\mathrm{K}(\mathrm{mmol} / \mathrm{L})$ & $3.9 \pm 0.6$ & $13.6 \pm 1.7$ & $24.5 \pm 2.1$ & $46.6 \pm 4.1$ \\
\hline$\overline{\mathrm{pH}}$ & $6.8 \pm 0.03$ & $6.74 \pm 0.03$ & $6.64 \pm 0.02$ & $6.37 \pm 0.04$ \\
\hline Lactate $(\mathrm{mmol} / \mathrm{L})$ & $3.6 \pm 0.4$ & $7.8 \pm 0.7$ & $17.2 \pm 2.5$ & $34.5 \pm 4.4$ \\
\hline Iron $(\mu \mathrm{mol} / \mathrm{L})$ & $3.8 \pm 0.9$ & $6.8 \pm 2.9$ & $7.6 \pm 1.6$ & $14.2 \pm 2.9$ \\
\hline Free hemoglobin (g/L) & $1.3 \pm 0.5$ & $1.5 \pm 0.8$ & $1.7 \pm 0.5$ & $3.0 \pm 2.1$ \\
\hline Percentage of irreversible deformed RBC & - & $8.4 \pm 1.6$ & $14.7 \pm 2.6$ & $29.9 \pm 4.0$ \\
\hline
\end{tabular}

interacts with nitric oxide (NO) leading to endothelial dysfunction and contributing to intravascular thrombosis, vasoconstriction, and leukocyte adhesion [37]. Finally, white blood cells present in the transfused RBC can increase hemolysis and potassium release, liberate oxygen radical, and increase erythrocyte alterations $[14,31]$.

\section{ICU patients may be especially susceptible to transfusion of older RBC}

The severity of illness of blood transfusion recipients may increase their susceptibility to the deleterious effects of $\mathrm{RBC}$ storage in several ways, making these concerns particularly relevant to critically ill patients. First, critically ill patients frequently have disease states that lead to impaired microcirculatory blood flow [38]. Second, their neutrophils may be primed by a trigger event (e.g., sepsis or trauma) and subsequent exposure to bioreactive substances of RBC unit may initiate enhanced activation of adherent leucocytes [39]. This hypothetical "two-hit" model was supported by a study that compared the transfusion of fresh or aged RBC into healthy rats with lipopolysaccharide pretreated rats. Transfusion of aged erythrocytes caused mild pulmonary inflammation but no coagulopathy in healthy rats, while it augmented lung injury by inducing coagulopathy in lipopolysaccharide pretreated rats. This difference was not found with the transfusion of fresh blood [39]. ICU patients are likely to undergo invasive mechanical ventilation (MV). Experimental data report an increase of transfusion related acute lung injury in mice receiving MV compared with those without MV. MV, particularly with injurious ventilator setting, induces lung injury and increase susceptibility of TRALI [40].

Finally, a "dose-effect" may exist and, because critically ill patients are especially susceptible to receiving multiple transfusions, this effect may increase their risk of developing adverse events [20,41]. In addition, receiving multiple transfusions will increase the risk that at least one of the RBC units transfused will be "old." In a retrospective study, Weinberg et al. found an independent increase in the risk of death when trauma patients received 6 or more $\mathrm{RBC}$ units with at least one $\mathrm{RBC}$ stored for $\geq 14$ days [16]. The same authors, in 1,647 trauma patients transfused within the first 24 hours postinjury, reported a higher independent risk of death only in patients who received 3 or more units stored for $\geq 14$ days $(\mathrm{RR}=1.57,95 \% \mathrm{CI} 1.14-2.15, p=0.01)$ [41].

\section{RBC storage and tissue oxygenation parameters}

In critically ill patients, $\mathrm{RBC}$ transfusion is commonly used to restore or increase tissue oxygenation. Different surrogate markers of tissue oxygenation have been used to explore the relationship between duration of $\mathrm{RBC}$ storage and their efficacy in restoring optimal tissue oxygenation. In 1993, Marik et al. were the first to report a harmful effect of duration of RBC storage on systemic and tissue oxygenation. In 23 mechanically ventilated septic patients, they prospectively demonstrated an inverse association between change in gastric intramucosal $\mathrm{pH}$ (reflecting oxygen uptake) and age of blood [42]. Nonetheless, this was a post-hoc analysis and such a relationship was not confirmed in three subsequent studies [43-45]. One of these negative studies was a doubleblind, randomized trial that compared the effect of RBC transfusion on tonometric indexes when RBC units were stored less than 5 days versus more than 20 days. This study did not show any difference between the study groups. Furthermore, there was no benefit from transfusing $\mathrm{RBC}$ whatever the age of blood possibly because of the defined transfusion target $(80-90 \mathrm{~g} / \mathrm{L})$ or because the poor sensitivity of the tonometric technique [44]. More recently, Sakr et al. did not find any impact of storage time on sublingual microvascular perfusion in a prospective, single-center, observational study conducted in 35 patients with severe sepsis and septic shock [45]. In the setting of severe traumatic brain injury, the impact of RBC storage duration on cerebral oxygenation assessed by brain tissue oxygen pressure $\left(\mathrm{PtiO}_{2}\right)$ was recently studied in an observational prospective study [46]. RBC stored for more than 19 days were unable to increase brain oxygenation, whereas fresh blood $(<19$ days) was effective [46]. In light of the contradictory findings of these five in vivo studies, it remains 
Table 2 Studies reporting a clinically harmful effect of prolonged RBC storage

\begin{tabular}{|c|c|c|c|c|c|c|c|}
\hline Authors & Year & Setting & $\mathbf{N}$ & $\begin{array}{l}\text { Study } \\
\text { design }\end{array}$ & $\begin{array}{l}\text { Main confounders used for } \\
\text { adjustment }\end{array}$ & Leukodepletion & Outcome and main results \\
\hline $\begin{array}{l}\text { Purdy et al. } \\
{[16]}\end{array}$ & 1997 & $\begin{array}{l}\text { ICU, severe } \\
\text { sepsis or septic } \\
\text { shock }\end{array}$ & 31 & $\begin{array}{l}\text { Retrospective } \\
\text { single-center }\end{array}$ & No & No & $\begin{array}{l}\text { Median of RBC storage was } \\
\text { lower in survivors }(17 \text { days) } \\
\text { than in nonsurvivors (median } \\
25 \text { days) }(p<0.0001)\end{array}$ \\
\hline $\begin{array}{l}\text { Zallen et al. } \\
\text { [28] }\end{array}$ & 1999 & $\begin{array}{l}\text { Trauma, } \geq 6 \\
\text { RBCs units in } \\
\text { the first } 12 \\
\text { hours post } \\
\text { injury }\end{array}$ & 63 & $\begin{array}{l}\text { Retrospective } \\
\text { single-center }\end{array}$ & $\begin{array}{l}\text { Patient age, serum lactate, base } \\
\text { deficit }\end{array}$ & No & $\begin{array}{l}\text { Mean age of RBC }>14 \text { days } \\
\text { associated with MOF (OR 1.16, } \\
\mathrm{Cl} 95 \%, 1.01-1.34, p=0.03)\end{array}$ \\
\hline $\begin{array}{l}\text { Vamvakas } \\
\text { et al. [23] }\end{array}$ & 1999 & Post-CABG & 416 & $\begin{array}{l}\text { Retrospective } \\
\text { single-center }\end{array}$ & $\begin{array}{l}\text { Chronic systemic illness, CABG } \\
\text { surgery type, IABP, intubation, } \\
\text { impaired consciousness, patient age, } \\
\text { bypass time, chest tube drainage, } \\
\text { admission WBC count }\end{array}$ & No & $\begin{array}{l}\text { Oldest blood was associated } \\
\text { with a higher risk of } \\
\text { pneumonia and/or wound } \\
\text { infection compared with fresh } \\
\text { blood (median of the mean } \\
\text { age of the oldest and second } \\
\text { oldest RBC units = } 21.6 \text { (range: } \\
\text { 4-41) days vs. } 13 \text { (range: } 2-39 \text { ) } \\
\text { days, } p=0.0002 \text { ) }\end{array}$ \\
\hline $\begin{array}{l}\text { Offner } \\
\text { et al. [26] }\end{array}$ & 2002 & $\begin{array}{l}\text { Trauma, } \geq 6 \\
\text { RBC units in the } \\
\text { first } 12 \text { hours } \\
\text { postinjury }\end{array}$ & 61 & $\begin{array}{l}\text { Prospective } \\
\text { single-center } \\
\text { observational }\end{array}$ & $\begin{array}{l}\text { Patient age, ISS, gender, mechanism } \\
\text { of injury }\end{array}$ & No & $\begin{array}{l}\text { Risk of major infectious } \\
\text { complications increased with } \\
\text { the number of RBC units }>14 \\
\text { days }(\mathrm{OR}=1.13,95 \% \mathrm{Cl}, 1.01 \text { - } \\
1.26, p=0.03)\end{array}$ \\
\hline $\begin{array}{l}\text { Keller et al. } \\
\text { [29] }\end{array}$ & 2002 & $\begin{array}{l}\text { Trauma with up } \\
\text { to } 4 \text { RBC units } \\
\text { in the first } 48 \\
\text { hours post } \\
\text { injury }\end{array}$ & 86 & $\begin{array}{l}\text { Retrospective } \\
\text { single-center }\end{array}$ & $\begin{array}{l}\text { ISS, requirement for surgery, volume } \\
\text { of } \mathrm{RBC} \text {, patient age }\end{array}$ & No & $\begin{array}{l}\text { Association between the } \\
\text { number of RBC }>14 \text { days and } \\
\text { hospital LOS }\end{array}$ \\
\hline $\begin{array}{l}\text { Leal-Noval } \\
\text { et al. [24] }\end{array}$ & 2003 & $\begin{array}{l}\text { Post-CABG or } \\
\text { valve surgery }\end{array}$ & 585 & $\begin{array}{l}\text { Prospective } \\
\text { single-center } \\
\text { observational }\end{array}$ & $\begin{array}{l}\text { Re-intubation, central nervous system } \\
\text { dysfunction, Apache II score, MV } \\
\text { duration }\end{array}$ & No & $\begin{array}{l}\text { Association between older } \\
\text { RBC ( }>28 \text { days) and the risk of } \\
\text { pneumonia (OR }=1.06,95 \% \\
\mathrm{Cl}, 1.01-1.11, p=0.018) \text { No } \\
\text { association with mortality }\end{array}$ \\
\hline $\begin{array}{l}\text { Murrell } \\
\text { et al. [30] }\end{array}$ & 2005 & Trauma & 275 & $\begin{array}{l}\text { Retrospective } \\
\text { single-center }\end{array}$ & $\begin{array}{l}\text { Patient age, ISS, leukodepletion } \\
\text { volume of RBC }\end{array}$ & $95 \%$ & $\begin{array}{l}\text { Association between older } \\
\text { blood and longer ICU and } \\
\text { hospital LOS (RR }=1.15,95 \% \\
\text { Cl, 1.11-1.2) No association } \\
\text { with mortality }\end{array}$ \\
\hline $\begin{array}{l}\text { Koch et al. } \\
{[18]}\end{array}$ & 2008 & $\begin{array}{l}\text { Post-CABG or } \\
\text { valve surgery }\end{array}$ & 6002 & $\begin{array}{l}\text { Retrospective } \\
\text { single-center }\end{array}$ & Baseline characteristics & Mixed & $\begin{array}{l}\text { Old blood }>14 \text { days was } \\
\text { associated with mortality, MV } \\
\text { duration, renal failure, } \\
\text { infections and MOF }\end{array}$ \\
\hline $\begin{array}{l}\text { Weinberg } \\
\text { et al. [17] }\end{array}$ & 2008 & $\begin{array}{l}\text { Trauma, } \geq 1 \\
\text { RBC unit in the } \\
\text { first } 24 \text { hours } \\
\text { post injury }\end{array}$ & 1813 & $\begin{array}{l}\text { Retrospective } \\
\text { single-center }\end{array}$ & $\begin{array}{l}\text { Patient age, gender, ISS, mechanism } \\
\text { of injury, volume of RBC, hospital LOS }\end{array}$ & Yes & $\begin{array}{l}\text { Transfusion } \geq 6 \text { RBC units of } \\
\text { RBC older } \geq 14 \text { days was } \\
\text { associated with higher } \\
\text { mortality }\end{array}$ \\
\hline $\begin{array}{l}\text { Weinberg } \\
\text { et al. [22] }\end{array}$ & 2008 & $\begin{array}{l}\text { Trauma without } \\
\text { RBC transfusion } \\
\text { in the first } 48 \\
\text { hours post } \\
\text { injury }\end{array}$ & 430 & $\begin{array}{l}\text { Retrospective } \\
\text { single-center }\end{array}$ & $\begin{array}{l}\text { Patient age, gender, ISS, presence of } \\
\text { thoracic injury, MV, volume of RBC }\end{array}$ & Yes & $\begin{array}{l}\mathrm{RBC} \geq 14 \text { days was associated } \\
\text { with mortality }(\mathrm{OR}=1.12,95 \% \\
\mathrm{Cl}: 1.02 \text { to } 1.23) \text {, renal failure } \\
(\mathrm{OR}=1.18,95 \% \mathrm{Cl}, 1.07-1.29) \\
\text { and pneumonia }(\mathrm{OR}=1.10, \\
95 \% \mathrm{Cl}, 1.04-1.17) \text { Not with } \\
\text { ARDS }\end{array}$ \\
\hline $\begin{array}{l}\text { Weinberg } \\
\text { et al. [41] }\end{array}$ & 2010 & $\begin{array}{l}\text { Trauma, } \geq 1 \text { RBC } \\
\text { unit for the first } \\
24 \text { hours }\end{array}$ & 1647 & $\begin{array}{l}\text { Retrospective } \\
\text { single-center }\end{array}$ & $\begin{array}{l}\text { Patient age, gender, ISS, mechanism } \\
\text { of injury, volume of RBC,FFP and } \\
\text { platelets, presence of head injury }\end{array}$ & Yes & $\begin{array}{l}3 \text { or more } \mathrm{RBC} \geq 14 \text { days } \\
\text { increased risk of death }(\mathrm{RR}= \\
1.57,95 \% \mathrm{Cl}, 1.14-2.15, p= \\
0.01)\end{array}$ \\
\hline $\begin{array}{l}\text { Spinella } \\
\text { et al. [20] }\end{array}$ & 2009 & $\begin{array}{l}\text { Trauma, } \geq 5 \mathrm{RBC} \\
\text { units }\end{array}$ & 202 & $\begin{array}{l}\text { Retrospective } \\
\text { single-center }\end{array}$ & $\begin{array}{l}\text { Patient age, cryoprecipitate, Glasgow } \\
\text { coma score, ISS }\end{array}$ & Mixed & $\begin{array}{l}\text { Association between } \mathrm{RBC}>21 \\
\text { days and DVT occurrence } \\
\text { Association between } \mathrm{RBC}>28 \\
\text { days and mortality (OR }=4 \\
95 \% \mathrm{Cl}, 1.34-11.61)\end{array}$ \\
\hline
\end{tabular}


Table 2 Studies reporting a clinically harmful effect of prolonged RBC storage (Continued)

\begin{tabular}{|c|c|c|c|c|c|c|c|}
\hline $\begin{array}{l}\text { Vandrome } \\
\text { et al. [27] }\end{array}$ & 2009 & Trauma & 487 & $\begin{array}{l}\text { Retrospective } \\
\text { single-center }\end{array}$ & $\begin{array}{l}\text { Patient age, gender, ISS, mechanism } \\
\text { of injury and MV time }\end{array}$ & Yes & $\begin{array}{l}\text { Risk of pneumonia higher in } \\
\text { patients transfused with } \mathrm{RBC} \\
\geq 14 \text { days ( } \mathrm{RR}=1.42,95 \% \mathrm{Cl} \text {, } \\
\text { 1.01-2.02) }\end{array}$ \\
\hline $\begin{array}{l}\text { Robinson } \\
\text { et al. [21] }\end{array}$ & 2010 & $\begin{array}{l}\text { Post- } \\
\text { percutaneous } \\
\text { coronary } \\
\text { intervention }\end{array}$ & 909 & $\begin{array}{l}\text { Retrospective } \\
\text { multi center }\end{array}$ & $\begin{array}{l}\text { Volume of RBC, procedures details, } \\
\text { demographic characteristics }\end{array}$ & NG & $\begin{array}{l}\text { Increased in age of the } \\
\text { youngest RBC was associated } \\
\text { with 30-day mortality (HR = } \\
1.02,95 \% \mathrm{Cl}, 1.18-1.34, p< \\
0.001)\end{array}$ \\
\hline $\begin{array}{l}\text { Eikelboom } \\
\text { et al. [19] }\end{array}$ & 2010 & $\begin{array}{l}\text { Acute } \\
\text { cardiovascular } \\
\text { disease }\end{array}$ & 4933 & $\begin{array}{l}\text { Prospective } \\
\text { single-center } \\
\text { observational }\end{array}$ & $\begin{array}{l}\text { Demographic characteristics, } \\
\text { comorbidities, clinical characteristics, } \\
\text { patient } A B O \text { group }\end{array}$ & Yes & $\begin{array}{l}\text { Hospital mortality higher } \\
\text { when the oldest } \mathrm{RBC}>31 \text { days } \\
\text { compared with } \mathrm{RBC}<10 \text { days } \\
(\mathrm{RR}=1.48,95 \% \mathrm{Cl}, 1.07-2.05)\end{array}$ \\
\hline $\begin{array}{l}\text { Andreasen } \\
\text { et al. [25] }\end{array}$ & 2011 & $\begin{array}{l}\text { Post-CABG or } \\
\text { valve surgery }\end{array}$ & 1748 & $\begin{array}{l}\text { Retrospective } \\
\text { multicenter }\end{array}$ & $\begin{array}{l}\text { Place of surgery, patient age gender, } \\
\text { BMI, preoperative } \mathrm{Hb} \text {, diabetes, } \\
\text { reoperation due to bleeding, use of } \\
\text { cardiopulmonary bypass, } \\
\text { concomitant valve surgery, } \\
\text { comorbidities, volume of RBC and } \\
\text { platelets units, ABO blood group }\end{array}$ & Mixed & $\begin{array}{l}\text { Higher risk of severe } \\
\text { postoperative infections (OR = } \\
2.5,95 \% \mathrm{Cl}, 1.2-4.2 \text { ) in patients } \\
\text { with } \mathrm{RBC} \text { exclusively } \geq 14 \text { days }\end{array}$ \\
\hline $\begin{array}{l}\text { Pettila } \\
\text { et al. [15] }\end{array}$ & 2011 & ICU & 757 & $\begin{array}{l}\text { Prospective } \\
\text { multicenter } \\
\text { observational }\end{array}$ & $\begin{array}{l}\text { Apache III score, leukodepletion } \\
\text { status, pre-ICU transfusion, cardiac } \\
\text { surgery, other transfused blood } \\
\text { components, pretransfusion Hb } \\
\text { preceding the first transfusion, } \\
\text { centers }\end{array}$ & $80 \%$ & $\begin{array}{l}\text { Oldest RBC associated with } \\
\text { longer LOS and higher } \\
\text { mortality }\end{array}$ \\
\hline $\begin{array}{l}\text { Juffermans } \\
\text { et al. [61] }\end{array}$ & 2012 & Trauma & 196 & $\begin{array}{l}\text { Retrospective } \\
\text { single-center }\end{array}$ & $\begin{array}{l}\text { ISS, head trauma, surgery, use for } \\
\text { SDD, volume of RBC and of platelets }\end{array}$ & Yes & $\begin{array}{l}\text { Patients with infections } \\
\text { received more old blood ( }>14 \\
\text { days) than patients without } \\
\text { infections ( } 8 \text { RBC units (range: } \\
2-16 \text { ) versus } 4 \text { RBC units } \\
\text { (range: } 2-8), p=0.02 \text { ) }\end{array}$ \\
\hline
\end{tabular}

$R B C$ red blood cells; ICU intensive care unit; LOS length of stay; $M V$ mechanical ventilation; DVT deep vein thrombosis; ARDS acute respiratory distress syndrome; CABG coronary artery bypass graft; MOF multi organ failure; IABP intra-aortic balloon pump; WBC White blood cells; ISS injury severity score; $A P A C H E$ II score Acute Physiology and Chronic Health Evaluation II score; RR relative risk; BMI body mass index; Hb hemoglobin; FFP fresh frozen plasma; SDD selective digestive decontamination, $N G$ not given.

controversial whether storage lesions affect the ability of $\mathrm{RBC}$ to modulate tissue oxygenation.

\section{RBC storage and clinical outcome - Review}

We searched with the PubMed database studies comparing clinical outcomes of critically ill patients receiving fresh or old blood. Excluding non-English language reports and studies conducted in pediatric patients, we identified 32 studies that examined the clinical effect of blood storage in trauma patients, ICU patients, and patients undergoing cardiac surgery or those with acute heart disease [15-30,41,47-59]. Eighteen of these studies reported a deleterious effect of increasing duration of RBC storage on clinically relevant outcomes [15-30,41] (Table 2), whereas 14 of these studies did not demonstrate any effect of prolonged RBC storage (Table 3) [47-59].

\section{Positive studies ICU patients}

Two of the 18 positive studies were conducted in ICU patients $[15,16]$. The first was retrospective and underpowered and was conducted in 31 patients with severe sepsis or septic shock, and without adjustment for confounding factors [16]. The second was a recent, prospective, multicenter, observational study conducted in 47 Australian and New Zealand ICUs. This study enrolled 757 patients and found a significant increase in ICU LOS and in-hospital mortality rate in patients receiving the oldest blood (median of age $=17.6$ days, range: 12.9-24.0) versus the freshest (median of age = 7.5 days, range: 5.7-9.0; OR $=2.01,95 \%$ confidence interval [CI]: 1.07-3.77). This effect was seen after adjustment for severity of illness (APACHE III score), number of transfusions, pre-ICU transfusions, freshfrozen plasma and platelet transfusions, leukodepletion status, pretransfusion hemoglobin concentration, clustering of study sites, and cardiac surgery [15]. Figure 2 illustrates the association between hospital mortality and maximum age of RBC found by these authors. However, in this observational study, patients received a mix of blood of different age (e.g., fresh and old units) and the statistical analysis could only use a surrogate of age of blood (fresh or old) received by the patients, by using the age of the oldest $R B C$ unit as the unit as representative of age of red cell transfused for statistical assessment of their effect. 
Table 3 Studies reporting no clinical effect of prolonged RBC storage

\begin{tabular}{|c|c|c|c|c|c|c|c|}
\hline Author & Year & Setting & $\mathrm{N}$ & Study design & Adjustment for confounders & Leukodepletion & Outcome and main results \\
\hline $\begin{array}{l}\text { Wasser } \\
\text { et al. [47] }\end{array}$ & 1989 & Post- CABG & 237 & $\begin{array}{l}\text { Single-center } \\
\text { randomized Cases: } \\
\text { RBC }<12 \text { hours; controls: } \\
\text { RBC stored for } 2 \text { to } 5 \\
\text { days }\end{array}$ & NA & No & $\begin{array}{l}\text { No difference in bleeding and } \\
\text { RBC transfusion requirement, } \\
\text { nonetheless the platelets } \\
\text { counts and thrombotest were } \\
\text { significantly less altered in the } \\
\text { study arm }\end{array}$ \\
\hline $\begin{array}{l}\text { Schulman } \\
\text { et al. [48] }\end{array}$ & 2002 & $\begin{array}{l}\text { Trauma, } \geq 2 \\
\text { RBC units }\end{array}$ & 17 & $\begin{array}{l}\text { Single-center } \\
\text { randomized pilot study } \\
\text { "Fresh group": RBC }<11 \\
\text { days; "Old group": RBC } \\
>20 \text { days }\end{array}$ & NA & Yes & $\begin{array}{l}\text { Mortality, infectious } \\
\text { complications, respiratory } \\
\text { failure }\end{array}$ \\
\hline $\begin{array}{l}\text { Vamvakas } \\
\text { et al. [49] }\end{array}$ & 2000 & Post- CABG & 268 & $\begin{array}{l}\text { Retrospective single- } \\
\text { center }\end{array}$ & $\begin{array}{l}\text { Gender, patient age, } \\
\text { comorbidities, type of CABG, } \\
\text { IABP, duration of anaesthesia, } \\
\text { time on bypass, other surgery, } \\
\text { repeated surgery, chest tube } \\
\text { drainage volume }\end{array}$ & No & $\begin{array}{l}\text { Post-operative ICU LOS, } \\
\text { hospital LOS and MV duration }\end{array}$ \\
\hline $\begin{array}{l}\text { Gajic et al. } \\
\text { [50] }\end{array}$ & 2004 & $\begin{array}{l}\text { ICU patients } \\
\text { with MV }\end{array}$ & 181 & $\begin{array}{l}\text { Retrospective single- } \\
\text { center }\end{array}$ & $\begin{array}{l}\text { APACHE III score, Tidal volume, } \\
\text { thrombocytopenia, massive } \\
\text { transfusion }\end{array}$ & $70 \%$ & $\begin{array}{l}\text { Median storage duration of } \\
\text { the oldest RBC unit = } 20.3 \\
\text { days (range: } 16-31 \text { ) in } \\
\text { absence of ALI versus } 20.1 \\
\text { days (range: } 16-27 \text { ) in } \\
\text { presence of ALI }\end{array}$ \\
\hline $\begin{array}{l}\text { Hebert } \\
\text { et al. [51] }\end{array}$ & 2005 & $\mathrm{ICU}$ & 57 & $\begin{array}{l}\text { Double-blind } \\
\text { multicenter, randomized } \\
\text { pilot study }\end{array}$ & $\begin{array}{l}\text { Comorbidities, major } \\
\text { diagnostic grouping, center }\end{array}$ & Yes & $\begin{array}{l}\text { Composite outcome } \\
\text { (mortality, nosocomial } \\
\text { infections, thrombotic events, } \\
\text { ischemic stroke) }\end{array}$ \\
\hline $\begin{array}{l}\text { Van de } \\
\text { Watering } \\
\text { et al. [52] }\end{array}$ & 2006 & $\begin{array}{l}\text { Post- CABG } \\
\text { RBC given } \\
\text { during } \\
\text { surgery and } \\
\text { for } 3 \text { days } \\
\text { post-surgery }\end{array}$ & 2732 & $\begin{array}{l}\text { Retrospective single- } \\
\text { center study Cases: RBC } \\
<18 \text { days Controls: } \\
\text { standard cares }\end{array}$ & $\begin{array}{l}\text { Year of surgery, volume of } \\
\text { transfusion, duration of } \\
\text { surgery, previous CABG, } \\
\text { number of distal anastomoses, } \\
\text { patient age, gender, Hb at } \\
\text { admission }\end{array}$ & No & $\begin{array}{l}\text { 30-day survival, hospital and } \\
\text { ICU LOS }\end{array}$ \\
\hline $\begin{array}{l}\text { Taylor } \\
\text { et al. [53] }\end{array}$ & 2006 & ICU & 449 & $\begin{array}{l}\text { Prospective single- } \\
\text { center observational }\end{array}$ & $\begin{array}{l}\text { Patient age, survival } \\
\text { probability }\end{array}$ & Mixed & $\begin{array}{l}\text { Nosocomial infection, } \\
\text { mortality, ICU and hospital } \\
\text { LOS }\end{array}$ \\
\hline $\begin{array}{l}\text { Gajic et al. } \\
\text { [54] }\end{array}$ & 2007 & ICU with ALI & 74 & $\begin{array}{l}\text { Prospective single- } \\
\text { center case-control } \\
\text { study }\end{array}$ & $\begin{array}{l}\text { Patients characteristic, } \\
\text { transfusion factors }\end{array}$ & NG & $\begin{array}{l}\text { Patients with ALI (median of } \\
\text { average RBC storage }=22.9 \\
\text { days (range: } 17-31 \text { ) versus } \\
22.9 \text { days (range: } 15-30 \text { ) in } \\
\text { controls ( } p=0.801 \text { ) }\end{array}$ \\
\hline $\begin{array}{l}\text { Yap et al. } \\
{[55]}\end{array}$ & 2008 & $\begin{array}{l}\text { Post-CABG } \\
\text { and valve } \\
\text { surgery, } \geq 2 \\
\text { RBC units }\end{array}$ & 670 & $\begin{array}{l}\text { Retrospective single- } \\
\text { center }\end{array}$ & $\begin{array}{l}\text { Pre-operative risk profile, } \\
\text { volume of RBC }\end{array}$ & $<5 \%$ & $\begin{array}{l}\text { Mortality, renal failure, } \\
\text { nosocomial pneumonia, ICU } \\
\text { LOS, MV duration }\end{array}$ \\
\hline $\begin{array}{l}\text { Van } \\
\text { Buskirk } \\
\text { et al. [56] }\end{array}$ & 2010 & ICU & 298 & $\begin{array}{l}\text { Retrospective single- } \\
\text { center }\end{array}$ & $\begin{array}{l}\text { Volume of RBC, patient age, } \\
\text { gender, severity at ICU } \\
\text { admission, admission } \\
\text { diagnosis }\end{array}$ & NG & $\begin{array}{l}\text { Transfusion complications, } \\
\text { change in SOFA score, ICU } \\
\text { LOS, mortality }\end{array}$ \\
\hline $\begin{array}{l}\text { Katsios } \\
\text { et al. [57] }\end{array}$ & 2011 & $\mathrm{ICU}$ & 126 & $\begin{array}{l}\text { Prospective single- } \\
\text { center observational }\end{array}$ & $\begin{array}{l}\text { History of previous DVT, } \\
\text { chronic dialysis, platelets } \\
\text { transfusion, requirement of } \\
\text { vasopressors }\end{array}$ & No & DVT \\
\hline $\begin{array}{l}{ }^{*} \text { Mckenny } \\
\text { et al. [58] }\end{array}$ & 2011 & $\begin{array}{l}\text { Post-cardiac } \\
\text { surgery }\end{array}$ & 1153 & $\begin{array}{l}\text { Retrospective single- } \\
\text { center }\end{array}$ & $\begin{array}{l}\text { Volume of RBC, baseline and } \\
\text { patient characteristics }\end{array}$ & Yes & $\begin{array}{l}\text { Early post-operative mortality, } \\
\text { post-operative MV }>72 \mathrm{~h} \text {, renal } \\
\text { failure, infections, } 30 \text { day } \\
\text { mortality, hospital mortality, } \\
\text { prolonged MV, new renal } \\
\text { failure, infectious } \\
\text { complications and ICU LOS }\end{array}$ \\
\hline $\begin{array}{l}\text { Van } \\
\text { Straten } \\
\text { et al. [59] }\end{array}$ & 2011 & $\begin{array}{l}\text { Post-CABG, } \\
\leq 10 \text { RBC } \\
\text { units }\end{array}$ & 3475 & $\begin{array}{l}\text { Retrospective single- } \\
\text { center }\end{array}$ & $\begin{array}{l}\text { Patient age, comorbidities, } \\
\text { redo cardiac surgery, pre- } \\
\text { operative } \mathrm{Hb} \text {, emergency }\end{array}$ & Yes & Mortality \\
\hline
\end{tabular}




\begin{tabular}{|c|c|c|c|c|c|c|}
\hline & & & & & $\begin{array}{l}\text { operation, perioperative MI, } \\
\text { Re-exploration, year of } \\
\text { operation, volume of RBC, FFP } \\
\text { and platelets }\end{array}$ & \\
\hline $\begin{array}{l}\text { Kor et al. } \\
{[62]}\end{array}$ & 2012 & $\begin{array}{l}\text { ICU patients } \\
\text { with MV }\end{array}$ & 100 & $\begin{array}{l}\text { Double-blind } \\
\text { randomized single- } \\
\text { center Cases: one fresh } \\
\text { RBC unit ( }<5 \text { days) } \\
\text { Control: one RBC unit of } \\
\text { standard practices }\end{array}$ & Yes & $\begin{array}{l}\text { Change in } \mathrm{PaO}_{2} / \mathrm{FiO}_{2} \text { ratio, in } \\
\text { peak and plateau airway } \\
\text { pressures, in markers of } \\
\text { immune status and in } \\
\text { coagulation }\end{array}$ \\
\hline
\end{tabular}

$N A$ not applicable; $R B C$ red blood cell; ICU intensive care unit; LOS length of stay; $M V$ mechanical ventilation; $D V T$ deep vein thrombosis; $A L I$ acute lung injury; CABG coronary artery bypass graft, APACHE III score Acute Physiology and Chronic Health Evaluation III score; Hb hemoglobin; MI myocardial infarction; FFP freshfrozen plasma; SOFA Sequential Organ Failure Assessment; NG not given. *Blood stored up 35 days and not 42 days.

\section{Cardiovascular patients}

In the setting of cardiac surgery or acute cardiovascular heart diseases, six positive studies have been published. Of these, two were prospective [19,24] and four were retrospective $[18,21,23,25]$. Their methods and their primary results are summarized in Table 2 . Three of them have reported an increased incidence of postoperative infections in patients who were transfused with the oldest blood [23-25]. The largest study was conducted by Koch et al. and included 6,002 patients undergoing coronary artery bypass graft (CABG) and/or valve surgery [18]. Patients who received exclusively "oldest" blood defined by a storage duration longer than 14 days had longer duration of mechanical ventilation (9.7\% vs. $5.6 \%$, $p<0.001)$, an increased incidence of sepsis ( $4 \%$ vs. $2.8 \%$, $p=0.01)$ and a higher 1-year mortality rate $(11 \%$ vs. $7.4 \%, p<0.001)$. Transfusion of older RBC also was independently associated with an increased risk-adjusted rate of a composite of serious adverse events (25.9\% vs. $22.4 \%, p=0.001$ ) [18]. Despite a large sample size, this study suffered important limitations, including a retrospective and single-center design, the use of an arbitrary cutoff point to define fresh and old blood, and the absence of adjustment for some important confounding factors [60]. Two other reports, however, considered patients with heart disease also found an impact of RBC storage on mortality $[19,21]$. One, a retrospective multicenter study reported that the age of the youngest RBC transfused within 10 days of percutaneous coronary intervention was significantly associated with 30-day mortality after adjustment for confounders $(\mathrm{HR}=1.02$, 95\% CI 1.18-1.34, $p<0.001$ ) [21]. The other found a linear relationship between the age of blood and mortality in 4,933 cardiovascular disease patients in acute care facilities [19].

\section{Trauma patients}

In trauma patients, ten studies have reported an impact of the storage duration on mortality and/or morbidity [17,20,22,26-30,41]. All were retrospective, single-center studies. Trauma patients transfused with blood older than 14 days appeared to have a higher risk of developing postinjury multiorgan failure [28], infectious complications [22,26,27,61], renal dysfunction [22], greater LOS [29], and higher mortality [17,22,41]. Regardless of the arbitrary cutoff of 14 days to define fresh and old

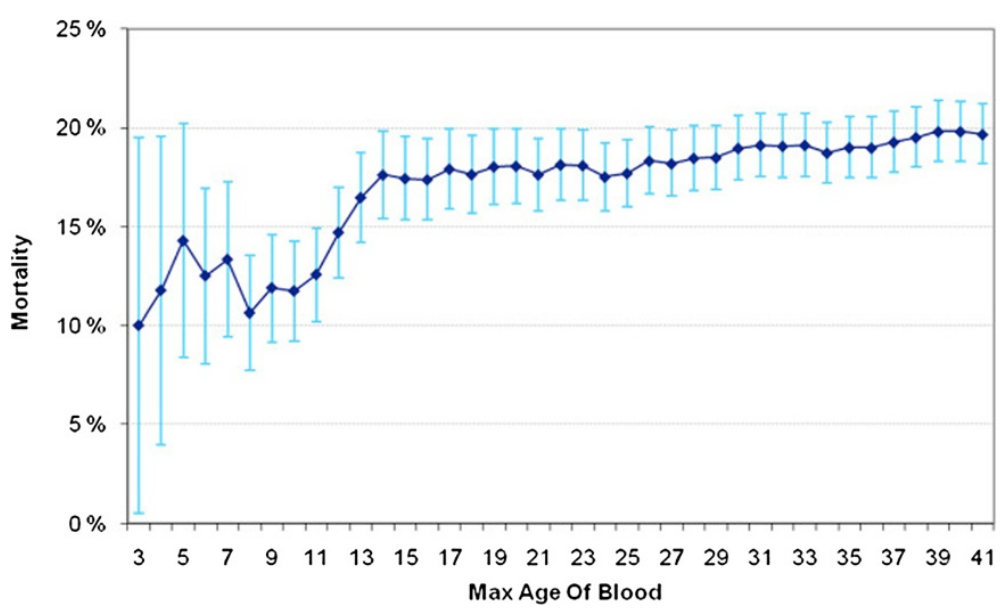

Figure 2 Hospital mortality (\%, 95\% confidence interval) according to maximum age of red blood cells (days) from Pettila et al. [15] with permission. 
blood, other studies have found an association between blood storage duration and hospital LOS [30], occurrence of deep vein thrombosis [20], and mortality [20,30]. Trauma patients are more likely to receive a "massive" transfusion and, as already mentioned, a dose effect of oldest blood may exist $[17,20,27,41]$.

Despite these positive observational studies, the evidence for a harmful effect of blood storage remains uncertain. This is because few of these reports adjusted for key confounding factors, including leukodepletion, volume of RBC transfused, the year of transfusion, and $\mathrm{ABO}$ type, and very few analyzed $\mathrm{RBC}$ age as a continuous variable [60]. None adjusted for all key confounders.

\section{Negative studies}

In contrast to the above-mentioned investigations, 14 studies did not find any relationship between blood storage duration and mortality, ICU and hospital LOS, duration of mechanical ventilation, acute lung injury, nosocomial infection, renal failure, or deep vein thrombosis occurrence (Table 3) [47-59,62].

\section{ICU patients}

One of the seven studies conducted in ICU patients was a double-blind, multicenter, randomized pilot study that enrolled 57 patients and compared a composite outcome, including mortality, between patients who received only blood of less than 8 days of age versus standard care. The small sample size $(n=57)$ likely contributed to an inconclusive result [51]. Taylor et al. reported an independent increase of nosocomial infection after blood transfusion in a prospective, observational study of 449 ICU patients but did not find any effect of age of RBC [53]. Three reports have especially studied the impact of $\mathrm{RBC}$ age on acute lung injury (ALI) or short-term pulmonary function in critically ill patients and did not find any difference $[50,54,62]$. Similarly, no effect of age of blood on deep vein thrombosis, ICU LOS, and mortality was found in two recent studies of ICU patients [56,57]. All of these studies were small and likely underpowered.

\section{Cardiovascular patients}

In postcardiac surgery patients, one of the six negative studies conducted more than 20 years ago was a singlecenter, randomized, controlled, blind trial that compared post-CABG bleeding in 237 patients who received either 2 units of freshly collected whole blood (fresher than 12 hours) followed by blood stored between 2 and 5 days or blood aged between 2 and 5 days. In this nonpragmatic study, there was no difference for bleeding and RBC transfusion requirements [47].

All the other negative, postcardiac surgery studies were retrospective and single center in design. Nonetheless, some had a large sample size [52,58,59]. For example, in a 2,732 patient cohort, Van de Watering et al. did not show any difference in 30-day survival, ICU, and hospital LOS between patients receiving fresh blood versus old blood, whatever the criteria to estimate the storage duration (only blood < or $>18$ days, the mean $\mathrm{RBC}$ storage time for each patient, the storage time of the youngest RBC transfused per patient, and the storage time of the oldest $\mathrm{RBC}$ transfused per patient) [52]. Similar results were reported by Yap et al. who did not find any association between $\mathrm{RBC}$ storage duration estimated by the mean age of $\mathrm{RBC}$ per patient, the oldest $\mathrm{RBC}$ unit and $\mathrm{RBC}$ stored longer than 30 days, and early postoperative mortality or morbidity in 670 post cardiac surgery patients [55]. Van Straten et al. also reported no association between the risk of early and late mortality and the age or the number of $\mathrm{RBC}$ units transfused during or within the 5 first days post-CABG [59].

\section{Trauma patients}

The only negative study in trauma patients was an underpowered $(\mathrm{n}=17)$ single-center, randomized trial that was conducted between 2000 and 2001. The study design required 15 compatible RBC units of both study arms to be available at randomization, which impacted the study feasibility [48].

\section{Need for randomized, controlled trials}

As outlined earlier, studies that evaluated the clinical impact of RBC storage in critically ill patients were 1) heterogeneous in outcomes, study design, population, threshold to differentiate fresh and old blood, and blood characteristics (leukodepleted or not, different storage medium, or storage medium not documented) and 2) often of insufficient quality in methodology (retrospective, observational, small sample size, no or limited inclusion of key confounding factors, and arbitrary cutoff for age of blood).

Their inherent limitations do not allow confirmation of the potential impact of duration of RBC storage on adverse effects or justify a change in current clinical transfusion practice. Two of the four randomized, controlled trials (RCTs) that evaluated clinical outcomes were very small and underpowered [48,51]. The third had a protocol based on the hypothesis that extremely fresh blood ( $<12$ hours) may contain functionally active platelets and coagulation factors and decrease postsurgery bleeding [47], and the most recent RCT only focused on the age and effect of a single RBC unit with mainly pulmonary complications as outcome [62].

Previous systematic reviews $[9-11,13]$ and a metaanalysis conducted in critically ill patients [63] have been inconclusive. Recently, in a meta-analysis, including 21 studies, Wang et al. concluded that older stored blood 


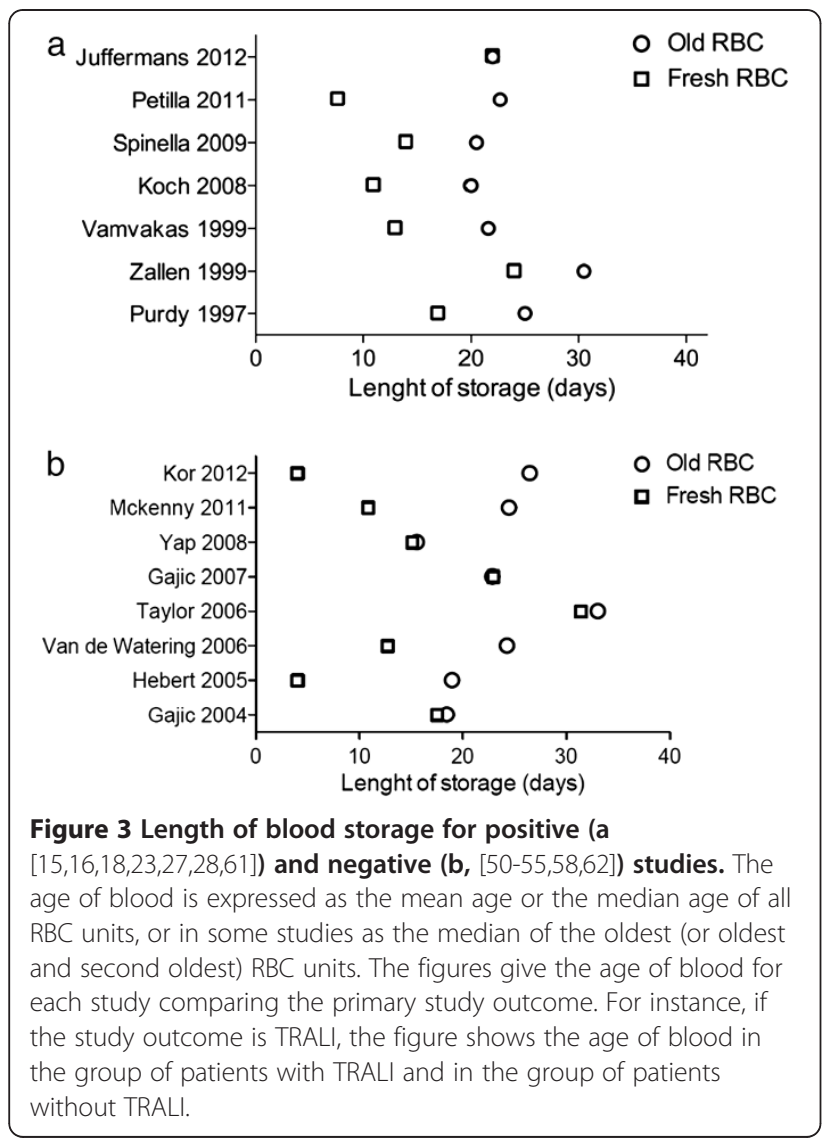

was associated with an estimated OR for death of 1.16 (95\% CI 1.07-1.24). However, some studies did not adjust the risk of death for important confounders, making their conclusions open to challenge [12]. In addition, definition of fresh and old blood remains based on observational studies and therefore unknown because of the heterogeneity of their results (Figures $3 a$ and $b$ ).

The above observations make a large, multicenter, RCT in critically ill patients of the greatest priority [64]. In this regard, several such multicenter RCTs are currently underway in adult critically ill patients (Table 4) [65].

The first such trial is the RECESS (Red Cell Storage Duration Study [RECESS]: NCT00991341) study. RECESS will randomize 1,434 cardiac surgical patients to receive either $\mathrm{RBC}$ stored 10 days or less or 21 days or more. However, its results could not be generalizable to any heterogeneous ICU population.

The Canadian ABLE study (Age of Blood Evaluation [ABLE] trial of the resuscitation of critically ill patients: ISRCTN44878718) is currently being conducted in ICU patients. It will enroll a total of 2,510 patients in Canada, France, and the United Kingdom [66]. The ABLE study will compare 90-day mortality between patients transfused with fresh RBC (defined as a storage duration $<8$ days) and patients transfused in accordance with standard practices [66]. Potential limitations of ABLE include 1) a sample size based on an estimated $25 \%$ relative risk reduction in the primary endpoint and 2) a design (fresh blood always fresher than 8 days) that seems unlikely to be reproducible always in future clinical practice.

The TRANSFUSE trial (ACTRN12612000453886) commences in 2012 in Australia and New Zealand. TRANSFUSE is a large (5,000 patients) pivotal, multicenter, randomized, controlled trial in critically ill patients to determine whether, compared with standard care, transfusion of the freshest available RBC decreases patient mortality. Completion is expected in 2015 and its findings are likely to guide blood transfusion policy in ICU patients.

\section{Conclusions}

Blood transfusion is a common therapeutic intervention in critically ill patients. Much scientific evidence, however, supports the occurrence of alterations in red cells over their storage time, and observational studies suggest that transfusion of older RBC may have important adverse clinical consequences, including mortality in this population. Nonetheless, making structural changes in

Table 4 Multicenter, randomized, clinical trials about blood storage in critically ill adults

\begin{tabular}{|c|c|c|c|c|c|c|}
\hline Authors or study name & Population & $\begin{array}{l}\text { Sample } \\
\text { size }\end{array}$ & Case criteria & $\begin{array}{l}\text { Controlled } \\
\text { criteria }\end{array}$ & Outcome & Status \\
\hline Hebert et al. [51] & ICU & 57 & $<8$ days & $\begin{array}{l}\text { Standard } \\
\text { practices }\end{array}$ & $\begin{array}{l}\text { Composite outcome* } \\
\text { (pilot study) }\end{array}$ & Achieved \\
\hline Aubron et al. [67] & ICU & 51 & $\begin{array}{l}\text { Freshest compatible } \\
\text { available RBC }\end{array}$ & $\begin{array}{l}\text { Standard } \\
\text { practices }\end{array}$ & Feasibility (pilot study) & Achieved \\
\hline${ }^{* *}$ RECESS (NCT00991341) & Post cardiac surgery & 1434 & $\leq 10$ days & $\geq 21$ days & Change in MODS & $\begin{array}{l}\text { In } \\
\text { progress }\end{array}$ \\
\hline${ }^{* *}$ ABLE (ISRCTN44878718) & ICU & 2510 & $<8$ days & $\begin{array}{l}\text { Standard } \\
\text { practices }\end{array}$ & 90-day mortality & $\begin{array}{l}\text { In } \\
\text { progress }\end{array}$ \\
\hline $\begin{array}{l}\text { **TRANSFUSE } \\
\text { (ACTRN12612000453886) }\end{array}$ & $\begin{array}{l}\text { ICU excluding } \\
\text { postcardiac surgery }\end{array}$ & 5000 & $\begin{array}{l}\text { Freshest compatible } \\
\text { available RBC }\end{array}$ & $\begin{array}{l}\text { Standard } \\
\text { practices }\end{array}$ & 90-day mortality & $\begin{array}{l}\text { In } \\
\text { progress }\end{array}$ \\
\hline
\end{tabular}

RECESS, Red Cell Storage Duration Study; ABLE, Age of Blood Evaluation; TRANSFUSE, STandaRd Issue TrANsfusion versuS Fresher red blood cell Use in intenSive carE; MODS multiorgan dysfunction score; **Indicates the trial is in progress.

*Included hospital mortality, serious nosocomial infections, thrombotic events with myocardial infarction and acute ischemic stroke. 
transfusion policy to deliver only fresh red cells to critically ill patients would have far-reaching logistics. The possibility that a clinically significant risk of older RBC transfusion exists and the possible benefits of making adjustments to transfusion policy can only be resolved by supporting and completing the current, pivotal, multicenter, double-blind RCTs. Until such trials are reported, any clinical practice change is premature.

\section{Abbreviations}

ICU: Intensive care unit; RBC: Red blood cells; LOS: Length of stay; ATP: Adenosine triphosphate; 2,3-DPG: 2,3-diphosphoglycerate; APACHE III: Acute Physiology Age Chronic Health Evaluation; CABG: Coronary artery bypass graft; RCT: Randomized controlled trials; ALl: Acute lung injury.

\section{Competing interests}

The authors declare they have no competing interests.

\section{Authors' contribution}

CA and RB conceived the review. CA executed the necessary search and wrote the first draft. RB, AN, and JC reviewed and modified the draft. CA and $\mathrm{RB}$ reviewed and completed the final version. All authors read and approved the final manuscript.

Received: 14 September 2012 Accepted: 1 December 2012

Published: 15 January 2013

\section{References}

1. Corwin HL, Surgenor SD, Gettinger A: Transfusion practice in the critically ill. Crit Care Med 2003, 31:S668-S671.

2. Corwin HL, Gettinger A, Pearl RG, Fink MP, Levy MM, Abraham E, Maclntyre NR, Shabot MM, Duh MS, Shapiro MJ: The CRIT Study: Anemia and blood transfusion in the critically ill-current clinical practice in the United States. Crit Care Med 2004, 32:39-52.

3. Vincent JL, Baron JF, Reinhart K, Gattinoni L, Thijs L, Webb A, MeierHellmann A, Nollet G, Peres-Bota D: Anemia and blood transfusion in critically ill patients. JAMA 2002, 288:1499-1507.

4. Blood Observational Study Investigators of ANZICS-Clinical Trials Group, Westbrook A, Pettilä V, Nichol A, Bailey MJ, Syres G, Murray L, Bellomo R, Wood E, Phillips LE, Street A, French C, Orford N, Santamaria J, Cooper DJ: Transfusion practice and guidelines in Australian and New Zealand intensive care units. Intensive Care Med 2010, 36:1138-1146.

5. Musallam KM, Tamim HM, Richards T, Spahn DR, Rosendaal FR, Habbal A Khreiss M, Dahdaleh FS, Khavandi K, Sfeir PM, et al: Preoperative anaemia and postoperative outcomes in non-cardiac surgery: a retrospective cohort study. Lancet 2011, 378:1396-1407.

6. Sabatine MS, Morrow DA, Giugliano RP, Burton PB, Murphy SA, McCabe $\mathrm{CH}$, Gibson CM, Braunwald E: Association of hemoglobin levels with clinical outcomes in acute coronary syndromes. Circulation 2005, 111:2042-2049.

7. Hebert PC, Wells G, Blajchman MA, Marshall J, Martin C, Pagliarello G, Tweeddale M, Schweitzer I, Yetisir E: A multicenter, randomized, controlled clinical trial of transfusion requirements in critical care. Transfusion Requirements in Critical Care Investigators, Canadian Critical Care Trials Group. N Engl I Med 1999, 340:409-417.

8. Marik PE, Corwin HL: Efficacy of red blood cell transfusion in the critically ill: a systematic review of the literature. Crit Care Med 2008, 36:2667-2674.

9. Triulzi DJ, Yazer MH: Clinical studies of the effect of blood storage on patient outcomes. Transfus Apher Sci 2010, 43:95-106.

10. Tinmouth A, Fergusson D, Yee IC, Hebert PC: Clinical consequences of red cell storage in the critically ill. Transfusion 2006, 46:2014-2027.

11. Lelubre C, Piagnerelli M, Vincent JL: Association between duration of storage of transfused red blood cells and morbidity and mortality in adult patients: myth or reality? Transfusion 2009, 49:1384-1394

12. Wang D, Sun J, Solomon SB, Klein HG, Natanson C: Transfusion of older stored blood and risk of death: a meta-analysis. Transfusion 2012. 52:1184-1195.

13. Zimrin $A B$, Hess JR: Current issues relating to the transfusion of stored red blood cells. Vox Sang 2009, 96:93-103.
14. Ho J, Sibbald WJ, Chin-Yee IH: Effects of storage on efficacy of red cell transfusion: when is it not safe? Crit Care Med 2003, 31:S687-S697.

15. Pettila V, Westbrook AJ, Nichol AD, Bailey MJ, Wood EM, Syres G, Phillips LE, Street A, French C, Murray L, et al: Age of red blood cells and mortality in the critically ill. Crit Care 2011, 15:R116.

16. Purdy FR, Tweeddale MG, Merrick PM: Association of mortality with age of blood transfused in septic ICU patients. Can J Anaesth 1997, 44:1256-1261

17. Weinberg JA, McGwin G Jr, Griffin RL, Huynh VQ, Cherry SA 3rd, Marques $M B$, Reiff DA, Kerby JD, Rue LW 3rd: Age of transfused blood: an independent predictor of mortality despite universal leukoreduction. J Trauma 2008, 65:279-282.

18. Koch CG, Li L, Sessler DI, Figueroa P, Hoeltge GA, Mihaljevic T, Blackstone EH: Duration of red-cell storage and complications after cardiac surgery. N Engl J Med 2008, 358:1229-1239.

19. Eikelboom JW, Cook RJ, Liu Y, Heddle NM: Duration of red cell storage before transfusion and in-hospital mortality. Am Heart J 2010, 159:737-743. e731

20. Spinella PC, Carroll CL, Staff I, Gross R, Mc Quay J, Keibel L, Wade CE, Holcomb JB: Duration of red blood cell storage is associated with increased incidence of deep vein thrombosis and in hospital mortality in patients with traumatic injuries. Crit Care 2009, 13:R151.

21. Robinson SD, Janssen C, Fretz EB, Berry B, Chase AJ, Siega AD, Carere RG, Fung A, Simkus G, Klinke WP, Hilton JD: Red blood cell storage duration and mortality in patients undergoing percutaneous coronary intervention. Am Heart J 2010, 159:876-881.

22. Weinberg JA, McGwin G Jr, Marques MB, Cherry SA 3rd, Reiff DA, Kerby JD, Rue LW 3rd: Transfusions in the less severely injured: does age of transfused blood affect outcomes? J Trauma 2008, 65:794-798

23. Vamvakas EC, Carven JH: Transfusion and postoperative pneumonia in coronary artery bypass graft surgery: effect of the length of storage of transfused red cells. Transfusion 1999, 39:701-710.

24. Leal-Noval SR, Jara-Lopez I, Garcia-Garmendia JL, Marin-Niebla A, HerruzoAviles A, Camacho-Larana P, Loscertales J: Influence of erythrocyte concentrate storage time on postsurgical morbidity in cardiac surgery patients. Anesthesiology 2003, 98:815-822.

25. Andreasen JJ, Dethlefsen C, Modrau IS, Baech J, Schonheyder HC, Moeller $J K$, Johnsen SP: Storage time of allogeneic red blood cells is associated with risk of severe postoperative infection after coronary artery bypass grafting. Eur J Cardiothorac Surg 2011, 39:329-334.

26. Offner PJ, Moore EE, Biffl WL, Johnson JL, Silliman CC: Increased rate of infection associated with transfusion of old blood after severe injury. Arch Surg 2002, 137:711-716. discussion 716-717.

27. Vandromme MJ, McGwin G Jr, Marques MB, Kerby JD, Rue LW 3rd, Weinberg JA: Transfusion and pneumonia in the trauma intensive care unit: an examination of the temporal relationship. J Trauma 2009, 67:97-101.

28. Zallen G, Offner PJ, Moore EE, Blackwell J, Ciesla DJ, Gabriel J, Denny C, Silliman CC: Age of transfused blood is an independent risk factor for postinjury multiple organ failure. Am J Surg 1999, 178:570-572.

29. Keller ME, Jean R, LaMorte WW, Millham F, Hirsch E: Effects of age of transfused blood on length of stay in trauma patients: a preliminary report. J Trauma 2002, 53:1023-1025.

30. Murrell Z, Haukoos JS, Putnam B, Klein SR: The effect of older blood on mortality, need for ICU care, and the length of ICU stay after major trauma. Am Surg 2005, 71:781-785.

31. Chin-Yee I, Arya N, d'Almeida MS: The red cell storage lesion and its implication for transfusion. Transfus Sci 1997, 18:447-458.

32. Wolfe $L C$ : The membrane and the lesions of storage in preserved red cells. Transfusion 1985, 25:185-203.

33. Card RT, Mohandas N, Perkins HA, Shohet SB: Deformability of stored red blood cells. Relationship to degree of packing. Transfusion 1982, 22:96-101.

34. Silliman CC, Moore EE, Kelher MR, Khan SY, Gellar L, Elzi DJ: Identification of lipids that accumulate during the routine storage of prestorage leukoreduced red blood cells and cause acute lung injury. Transfusion 2011, 51:2549-2554.

35. Karam O, Tucci M, Toledano BJ, Robitaille N, Cousineau J, Thibault L, Lacroix J, Le Deist F: Length of storage and in vitro immunomodulation induced by prestorage leukoreduced red blood cells. Transfusion 2009, 49:2326-2334. 
36. Hod EA, Brittenham GM, Billote GB, Francis RO, Ginzburg YZ, Hendrickson JE, Jhang J, Schwartz J, Sharma S, Sheth S, et al: Transfusion of human volunteers with older, stored red blood cells produces extravascular hemolysis and circulating non-transferrin-bound iron. Blood 2011, 118:6675-6682.

37. Lee JS, Gladwin MT: Bad blood: the risks of red cell storage. Nat Med 2010, 16:381-382.

38. Chierego M, Verdant C, De Backer D: Microcirculatory alterations in critically ill patients. Minerva Anesthesio/ 2006, 72:199-205.

39. Vlaar AP, Hofstra JJ, Levi M, Kulik W, Nieuwland R, Tool AT, Schultz MJ, de Korte D, Juffermans NP: Supernatant of aged erythrocytes causes lung inflammation and coagulopathy in a "two-hit" in vivo syngeneic transfusion model. Anesthesiology 2010, 113:92-103.

40. Vlaar AP, Wolthuis EK, Hofstra JJ, Roelofs JJ, Boon L, Schultz MJ, Lutter R, Juffermans NP: Mechanical ventilation aggravates transfusion-related acute lung injury induced by MHC-I class antibodies. Intensive Care Med 2010, 36:879-887.

41. Weinberg JA, McGwin G Jr, Vandromme MJ, Marques MB, Melton SM, Reiff DA, Kerby JD, Rue LW 3rd: Duration of red cell storage influences mortality after trauma. J Trauma 2010, 69:1427-1432.

42. Marik PE, Sibbald WJ: Effect of stored-blood transfusion on oxygen delivery in patients with sepsis. JAMA 1993, 269:3024-3029.

43. Fernandes C Jr, Akamine N, De Marco FV, De Souza JA, Lagudis S, Knobel E: Red blood cell transfusion does not increase oxygen consumption in critically ill septic patients. Crit Care 2001, 5:362-367.

44. Walsh TS, McArdle F, McLellan SA, Maciver C, Maginnis M, Prescott RJ, McClelland DB: Does the storage time of transfused red blood cells influence regional or global indexes of tissue oxygenation in anemic critically ill patients? Crit Care Med 2004, 32:364-371

45. Sakr Y, Chierego M, Piagnerelli M, Verdant C, Dubois MJ, Koch M, Creteur J, Gullo A, Vincent JL, De Backer D: Microvascular response to red blood cell transfusion in patients with severe sepsis. Crit Care Med 2007, 35:1639-1644

46. Leal-Noval SR, Munoz-Gomez M, Arellano-Orden V, Marin-Caballos A Amaya-Villar R, Marin A, Puppo-Moreno A, Ferrandiz-Millon C, FloresCordero JM, Murillo-Cabezas F: Impact of age of transfused blood on cerebral oxygenation in male patients with severe traumatic brain injury. Crit Care Med 2008, 36:1290-1296.

47. Wasser MN, Houbiers JG, D'Amaro J, Hermans J, Huysmans HA, van Konijnenburg GC, Brand A: The effect of fresh versus stored blood on post-operative bleeding after coronary bypass surgery: a prospective randomized study. Br J Haematol 1989, 72:81-84.

48. Schulman Cl, Nathe K, Brown M, Cohn SM: Impact of age of transfused blood in the trauma patient. J Trauma 2002, 52:1224-1225.

49. Vamvakas EC, Carven JH: Length of storage of transfused red cells and postoperative morbidity in patients undergoing coronary artery bypass graft surgery. Transfusion 2000, 40:101-109.

50. Gajic O, Rana R, Mendez JL, Rickman OB, Lymp JF, Hubmayr RD, Moore SB: Acute lung injury after blood transfusion in mechanically ventilated patients. Transfusion 2004, 44:1468-1474.

51. Hebert PC, Chin-Yee I, Fergusson D, Blajchman M, Martineau R, Clinch J, Olberg B: A pilot trial evaluating the clinical effects of prolonged storage of red cells. Anesth Analg 2005, 100:1433-1438. table of contents.

52. Van de Watering L, Lorinser J, Versteegh M, Westendord R, Brand A: Effects of storage time of red blood cell transfusions on the prognosis of coronary artery bypass graft patients. Transfusion 2006, 46:1712-1718.

53. Taylor RW, O'Brien J, Trottier SJ, Manganaro L, Cytron M, Lesko MF, Arnzen K, Cappadoro C, Fu M, Plisco MS, et al: Red blood cell transfusions and nosocomial infections in critically ill patients. Crit Care Med 2006, 34:2302-2308.

54. Gajic O, Rana R, Winters JL, Yilmaz M, Mendez JL, Rickman OB, O'Byrne MM, Evenson LK, Malinchoc M, DeGoey SR, et al: Transfusion-related acute lung injury in the critically ill: prospective nested case-control study. Am J Respir Crit Care Med 2007, 176:886-891.

55. Yap CH, Lau L, Krishnaswamy M, Gaskell M, Yii M: Age of transfused red cells and early outcomes after cardiac surgery. Ann Thorac Surg 2008, 86:554-559.

56. Van Buskirk CM, Thakur SJ, Murray DL, Bryant SC, Winters JL, Stubbs JR, Gajic $\mathrm{O}$ : Red blood cell storage age has no impact on clinical outcome in critically ill patients. Transfusion 2009, 49. SupplementS3.
57. Katsios C, Griffith L, Spinella P, Lacroix J, Crowther M, Hebert P, Meade M, Geerts W, Rabbat C, Cook D: Red blood cell transfusion and increased length of storage are not associated with deep vein thrombosis in medical and surgical critically ill patients: a prospective observational cohort study. Crit Care 2011, 15:R263.

58. McKenny M, Ryan T, Tate H, Graham B, Young VK, Dowd N: Age of transfused blood is not associated with increased postoperative adverse outcome after cardiac surgery. Br J Anaesth 2011, 106:643-649.

59. Van Straten AH, Soliman Hamad MA, van Zundert AA, Martens EJ, ter Woorst JF, de Wolf AM, Scharnhorst V: Effect of duration of red blood cell storage on early and late mortality after coronary artery bypass grafting. J Thorac Cardiovasc Surg 2011, 141:231-237.

60. Van de Watering L: Pitfalls in the current published observational literature on the effects of red blood cell storage. Transfusion 2011, 51:1847-1854.

61. Juffermans NP, Vlaar AP, Prins DJ, Goslings JC, Binnekade JM: The age of red blood cells is associated with bacterial infections in critically ill trauma patients. Blood Transfus 2012, 10:290-295.

62. Kor DJ, Gajic O: Blood product transfusion in the critical care setting. Curr Opin Crit Care 2012, 16:309-316.

63. Vamvakas EC: Meta-analysis of clinical studies of the purported deleterious effects of "old" (versus "fresh") red blood cells: are we at equipoise? Transfusion 2010, 50:600-610.

64. Aubron C, Carteaux G, Cooper DJ: Why we must "TRANSFUSE.". Crit Care Resusc 2011, 13:67-68.

65. Steiner ME, Assmann SF, Levy JH, Marshall J, Pulkrabek S, Sloan SR, Triulzi D, Stowell CP: Addressing the question of the effect of RBC storage on clinical outcomes: the Red Cell Storage Duration Study (RECESS) (Section 7). Transfus Apher Sci, 43:107-116.

66. Lacroix J, Hebert P, Fergusson D, Tinmouth A, Blajchman MA, Callum J, Cook D, Marshall JC, Mclntyre L, Turgeon AF: The Age of Blood Evaluation (ABLE) randomized controlled trial: study design. Transfus Med Rev 2011, 25:197-205.

67. Aubron C, Syres G, Nichol A, Bailey M, Board J, Magrin G, Murray L, Presneill J, Sutton J, Vallance S, Morrison S, Bellomo B, Cooper DJ: A pilot feasibility trial of allocation of freshest available red blood cells versus standard care in critically ill patients. Transfusion 2012, 52:1196-1202.

doi:10.1186/2110-5820-3-2

Cite this article as: Aubron et al:: Age of red blood cells and transfusion in critically ill patients. Annals of Intensive Care 2013 3:2.

\section{Submit your manuscript to a SpringerOpen ${ }^{\odot}$ journal and benefit from:}

- Convenient online submission

- Rigorous peer review

- Immediate publication on acceptance

- Open access: articles freely available online

- High visibility within the field

- Retaining the copyright to your article

Submit your next manuscript at $>$ springeropen.com 